This item was submitted to Loughborough's Research Repository by the author.

Items in Figshare are protected by copyright, with all rights reserved, unless otherwise indicated.

\title{
Uv absorption inmetal decorated boron nitride flakes: A theoretical analysis of excited states
}

PLEASE CITE THE PUBLISHED VERSION

https://doi.org/10.1080/00268976.2017.1324646

\section{PUBLISHER}

(c) Taylor \& Francis

\section{VERSION}

AM (Accepted Manuscript)

\section{PUBLISHER STATEMENT}

This work is made available according to the conditions of the Creative Commons Attribution-NonCommercialNoDerivatives 4.0 International (CC BY-NC-ND 4.0) licence. Full details of this licence are available at: https://creativecommons.org/licenses/by-nc-nd/4.0/

\section{LICENCE}

CC BY-NC-ND 4.0

\section{REPOSITORY RECORD}

Chopra, Siddheshwar, and Felix Plasser. 2019. "Uv Absorption Inmetal Decorated Boron Nitride Flakes: A Theoretical Analysis of Excited States". figshare. https://hdl.handle.net/2134/32231. 


\title{
UV Absorption in metal decorated Boron nitride flakes: A theoretical analysis of excited states
}

\author{
Siddheshwar Chopra ${ }^{\mathrm{a},}$, Felix Plasser ${ }^{\mathrm{b}}$ \\ *Department of Physics, AIAS, Amity University, Noida, India \\ *Institute for Theoretical Chemistry, Faculty of Chemistry, University of Vienna, \\ Währingerstr. 17, 1090 Vienna, Austria
}

\begin{abstract}
The excited states of single metal atom $(\mathrm{X}=\mathrm{Co}, \mathrm{Al}$ and $\mathrm{Cu})$ doped boron nitride flake (MBNF) $\mathrm{B}_{15} \mathrm{~N}_{14} \mathrm{H}_{14}-\mathrm{X}$ and pristine boron nitride $\left(\mathrm{B}_{15} \mathrm{~N}_{15} \mathrm{H}_{14}\right)$ are studied by time-dependent density functional theory. The immediate effect of metal doping is a red shift of the onset of absorption from about 220 $\mathrm{nm}$ for pristine BNF to above $300 \mathrm{~nm}$ for all metal doped variants with the biggest effect for MBNFCo, which shows appreciable intensity even above $400 \mathrm{~nm}$. These energy shifts are analyzed by detailed wavefunction analysis protocols using visualization methods, such as the natural transition orbital analysis and electron-hole correlation plots, as well as quantitative analysis of the exciton size and electron-hole populations. The analysis shows that the $\mathrm{Co}$ and $\mathrm{Cu}$ atoms provide strong contributions to the relevant states whereas the aluminum atom is only involved to a lesser extent.
\end{abstract}

Keywords: boron-nitride; TDDFT; excited state analysis; NTO; charge transfer

\section{Introduction}

Boron nitride materials are an active area of research due to their unique properties like high hardness and excellent chemical and thermal stabilities, which make them suitable in many potential applications like hydrogen and carbon dioxide storage media, sensing applications, piezoelectric and nanoelectronic devices ${ }^{1-5}$. Noticeably, there have been a few studies devoted to the optical properties of boron nitride nanoribbons (BNNRs) and nanotubes (BNNTs) ${ }^{6,7}$. It has been experimentally shown that boron nitride nanosheets can be used as deep-UV photodetectors 8. Carbon doping has been tested to tune the optical response of boron nitride nanodots 9 Recently, boron nitride structures have been investigated for the purpose of hydrogen storage ${ }^{10}$, where the addition of metal nanoparticles like platinum was found to boost the hydrogen storage capacity ${ }^{11}$. It is also known that the pure boron nitride systems do not bind hydrogen

*Corresponding author. E-mail address: schopra1@amity.edu (Siddheshwar Chopra) 
substantially $^{12}$. There are reports ${ }^{13,14}$ on the study of electronic and magnetic properties of single transition metal atom doped BNNRs which demonstrate their potential applications in spintronics and magnetic data storage. In our recent report ${ }^{15}$, we have implemented the density functional theory (DFT) and time-dependent density functional theory (TDDFT) based calculations for the study of ground state properties and photo-absorption of boron nitride flakes (BNFs) both in pure and single metal atom doped forms, replacing a nitrogen atom by the metal. The metal atoms used were $\mathrm{Ni}^{+}, \mathrm{Fe}^{+}, \mathrm{Co}, \mathrm{Cr}^{+}, \mathrm{Cu}$ and $\mathrm{Al}$. It was reported that the electronic gap of pure BNF shifts from insulating to semiconducting nature on single metal atom doping, along with the red shift in the absorption wavelengths from ultraviolet to visible region. So far, no experimental reference data on these systems are available. Following Ref. 15, the same systems are studied here but enhanced insight into the electronic wavefunctions involved is provided through extended analysis tools.

Time-dependent linear response density functional theory (TDDFT) has been commonly used to describe the electronic excitations of molecular many-particle systems ${ }^{16-19}$. In the TDDFT framework, electronic excitation energies and oscillator strengths of molecular systems are easily calculated. However, the more intricate details of the resulting response functions are often ignored or analyzed only superficially in the discussions. An analysis of excited states is particularly challenging in the case of extended molecular systems, as two contradicting viewpoints come into play for these systems ${ }^{20-22}$ : the molecular orbital (MO) and exciton quasiparticle representations. The former is needed to understand molecular details and to visualize the general state character $\left(\pi \pi^{*}, n \pi^{*}\right.$ etc.) whereas the latter provides more immediate insight into electron-hole correlations in larger systems. Recently, an extensive analysis framework was developed that allows to bridge between these concepts ${ }^{23-26}$. The central concept of these analysis strategies is a two-body exciton wavefunction, which is constructed from the transition density matrix (1TDM). Starting from this concept, a wide range of visual and quantitative analysis methods can be defined. In the following, we start by applying the popular concept of the natural transition orbital transformation (NTO ${ }^{24,27}$. The NTOs generally provide a more compact representation as opposed to the Hartree-Fock orbitals and are a convenient method to visualize the excitation hole and the excited electron. We proceed by computing electron-hole correlation plots ${ }^{23,28-30}$ to provide a more compact description of the different excitation processes. Finally, two quantitative analysis methods are explored: an electron-hole population analysis and the computation of exciton sizes. Application of these methods does not 
only provide detailed insight into the excited states of MBNFs but also allows studying the interrelations between the analysis methods. Recently one of the authors has employed similar transition density matrix based analysis to describe the excited states in graphene nanoribbons (GNR), both in pure and metal doped forms ${ }^{31}$. The present study supersedes this previous work by applying a more extended set of analysis methods and by performing a more fine-grained analysis of the electron-hole distributions.

\section{Methodology}

Structures considered in this study are the boron nitride flakes (BNFs) both in pure $\left(\mathrm{B}_{15} \mathrm{~N}_{15} \mathrm{H}_{14}\right)$ and neutral single-metal-atom doped $\left(\mathrm{B}_{15} \mathrm{~N}_{14} \mathrm{H}_{14}-\mathrm{X}\right.$, where $\mathrm{X}=\mathrm{Al}, \mathrm{Co}$, and $\left.\mathrm{Cu}\right)$ forms, from our previous study ${ }^{15}$ (Fig.1). All the calculations were performed with the Firefly version 8.1.0, build number 8800 program code ${ }^{32}$. TDDFT results at B3LYP/6-31+G $(\mathrm{d}, \mathrm{p})$ level of theory under Tamm-Dancoff approximation, for the single-photon absorption were used. The lowest 100 singlet excited states were studied. Analysis of excited state wavefunctions was performed by means of the TheoDORE software package ${ }^{33}$ using the cclib library for data parsing ${ }^{34}$ and the Jmol package for plotting ${ }^{35}$.

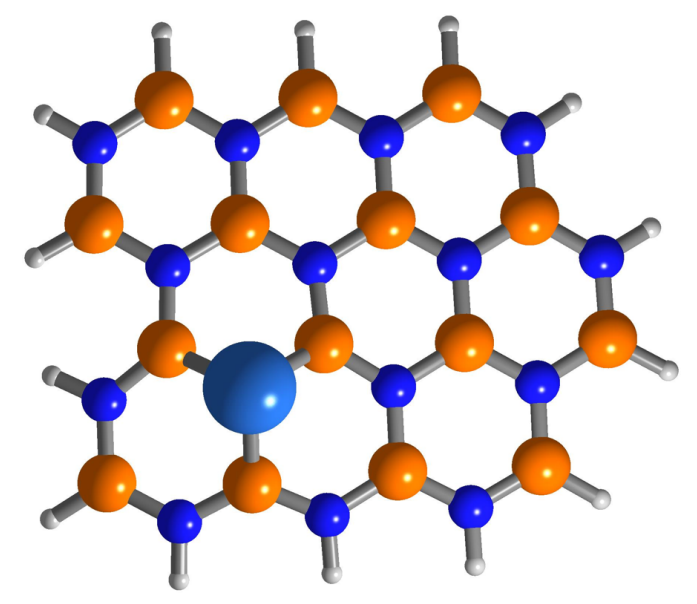

Fig 1. Metal doped $\left(\mathrm{B}_{15} \mathrm{~N}_{14} \mathrm{H}_{14}-\mathrm{X}\right) \mathrm{BNF}$ showing the common doping site for all the MBNF; where orange, blue, and grey colored atoms denote boron, nitrogen and hydrogen atoms. The large blue atom denotes the dopant metal atom. 


\section{Results and Discussion}

\subsection{Absorption strengths}

The computed stick spectra for UV/Vis absorption are presented in Fig. 2. This figure reflects the results given in a recent study by one of the authors ${ }^{15}$ : Metal doping produces a general red shift in the absorption spectrum and a reduction of the oscillator strengths. Both effects are strongest for MBNF-Co. Due to the large number of similar excitations in these systems it is not possible to provide a detailed description of all these states. Therefore, only some representative states are chosen for a more detailed analysis. This is done on the basis of higher oscillator strengths or for covering a higher absorption range. The oscillator strengths $f$ of these states are listed in Table 1. Oscillator strengths $(f)$ were determined using Casida equations ${ }^{17,18,36}$, as implemented in the FIREFLY code.
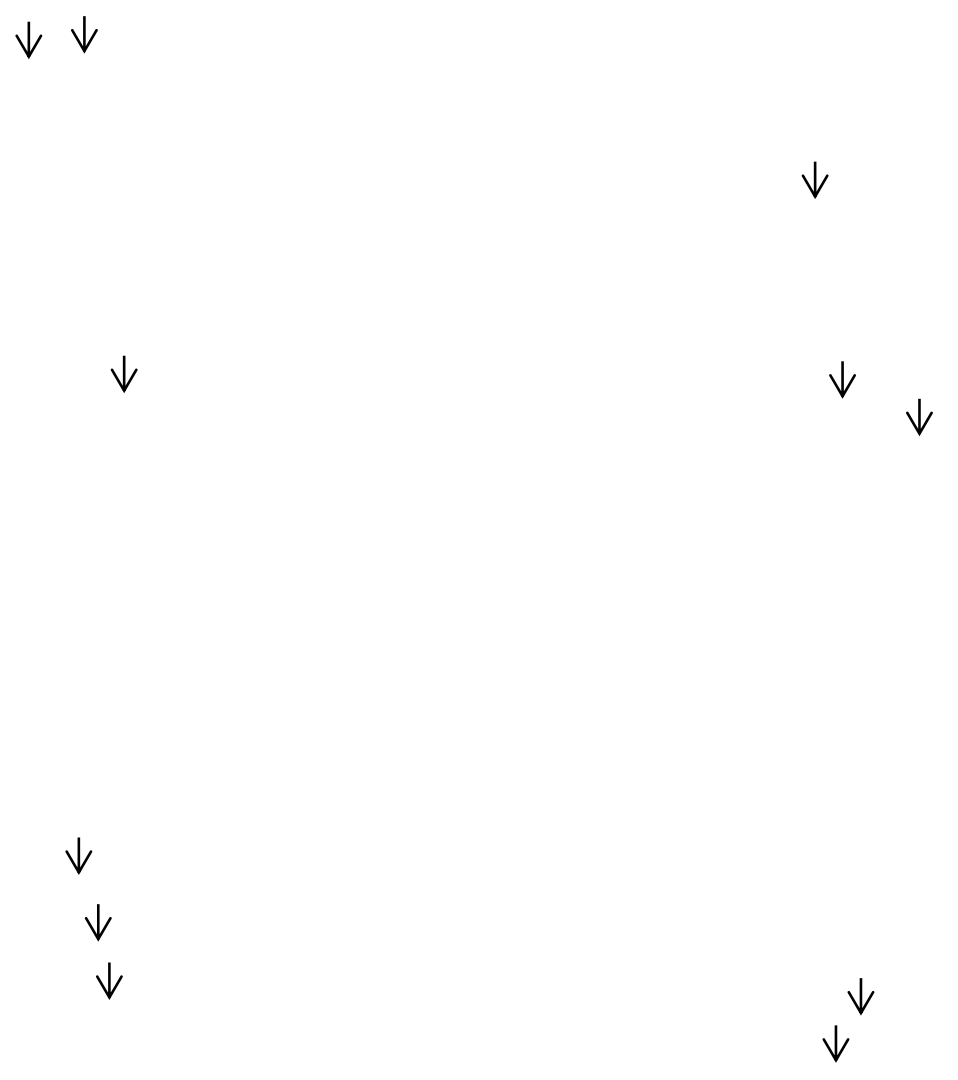

Fig. 2 Plots of Oscillator strength vs Excitation wavelength for all the BNFs. The arrow marks represent the transitions which are discussed in subsequent sections. 
Table 1. Oscillator strengths $(f)$ between the ground and $n^{\text {th }}$ excited state of all BNFs, are listed

\begin{tabular}{|c|c|cc|}
\hline \multirow{2}{*}{ BNF } & State & \multicolumn{2}{|c|}{$\mathbf{6 - 3 1 + G}(\mathbf{d}, \mathbf{p})$} \\
\cline { 3 - 4 } & transition & $\boldsymbol{\lambda ( \mathbf { n m } )}$ & $\boldsymbol{f}$ \\
\hline \multirow{4}{*}{ Pure } & $\mathrm{S}_{0} \rightarrow \mathrm{S}_{2}$ & $\mathbf{2 1 8}$ & 0.11 \\
& $\mathrm{~S}_{0} \rightarrow \mathrm{S}_{14}$ & $\mathbf{1 9 4}$ & $\mathbf{0 . 3 7}$ \\
& $\mathrm{S}_{0} \rightarrow \mathrm{S}_{94}$ & 159 & 0.37 \\
\hline \multirow{5}{*}{ MBNF-Al } & $\mathrm{S}_{0} \rightarrow \mathrm{S}_{51}$ & 208 & 0.08 \\
& $\mathrm{~S}_{0} \rightarrow \mathrm{S}_{61}$ & 201 & 0.13 \\
& $\mathrm{~S}_{0} \rightarrow \mathrm{S}_{77}$ & 192 & 0.18 \\
\hline & $\mathrm{S}_{0} \rightarrow \mathrm{S}_{7}$ & 411 & 0.02 \\
MBNF-Co & $\mathrm{S}_{0} \rightarrow \mathrm{S}_{9}$ & 399 & 0.03 \\
& $\mathrm{~S}_{0} \rightarrow \mathrm{S}_{69}$ & 214 & 0.07 \\
& $\mathrm{~S}_{0} \rightarrow \mathrm{S}_{96}$ & 200 & 0.03 \\
\hline \multirow{3}{*}{ MBNF-Cu } & $\mathrm{S}_{0} \rightarrow \mathrm{S}_{1}$ & 511 & 0.01 \\
& $\mathrm{~S}_{0} \rightarrow \mathrm{S}_{17}$ & 248 & 0.08 \\
& $\mathrm{~S}_{0} \rightarrow \mathrm{S}_{55}$ & 203 & 0.10 \\
& $\mathrm{~S}_{0} \rightarrow \mathrm{S}_{84}$ & 187 & 0.25 \\
\hline
\end{tabular}

\subsection{Natural Transition Orbitals (NTOs)}

To provide a compact description of the excitation process, the natural transition orbital (NTO) representation is chosen. The NTOs are constructed through a singular value (SV) decomposition $9,23,27$ :

$$
\mathbf{D}=\mathbf{U} \operatorname{diag}\left(\sqrt{\lambda_{1}}, \sqrt{\lambda_{2}}, \ldots\right) \mathbf{V}^{\mathrm{T}}
$$

Here $\mathbf{D}$ is the transition density matrix, constructed from TDDFT response vector. $\mathbf{U}$ and $\mathbf{V}$ are the unitary matrices describing the hole and particle orbitals. The $\lambda_{i}(\leq 1)$ values denote the weights of the respective configurations.

Fig. 3 illustrates the NTOs corresponding to the dominant singlet transitions $\left(S_{0} \rightarrow S_{n}\right)$ of all the structures along with the corresponding weights, $\lambda_{\mathrm{i}}$. The singlet excitations are described by single or multiple set of NTOs each (with $\lambda_{\mathrm{i}}$ ), as shown in Fig. 3. The extent of electron delocalization in pristine BNF is seen from Fig. 3, where the exciton wave function in the hole NTOs is concentrated primarily on the electron-rich nitrogen atoms spreading almost entirely on the BNF, and in the particle NTOs (upon excitation) it gets accumulated on the electron-deficient boron atoms almost entirely. But this picture of electron delocalization changes on doping.

In MBNF-Al, the electron delocalization enhances with electron density moving away from nitrogen (in hole NTOs) and builds up on the boron, aluminium and hydrogen atoms (in particle 
Fig. 3 Natural transition orbitals (NTOs) for the dominant excitations of all the BNFs, a) Pure, b) MBNF-Al, c) MBNF-Co and d) MBNF-Cu. The values in brackets represent the weigths $\left(\lambda_{i}\right)$ of respective NTOs. The arrow indicates the location of single metal atom in MBNFs. Pink and blue atoms denote boron and nitrogen atoms.

NTOs) spanning a larger area. In MBNF-Co, the electron cloud is concentrated on or around the cobalt and boron atoms and moves around insignificantly in the particle NTOs $\left(\lambda_{\mathrm{i}}=0.88\right)$. Also, 
in NTOs with $\lambda_{\mathrm{i}}=0.67$, the electron density moves from cobalt and nitrogen atoms, towards the boron atoms. Finally, in MBNF-Cu, the exciton wave function is concentrated on and around the copper atom and moves towards the terminal hydrogens and boron atoms on absorption. In our previous report ${ }^{31}$, the electron delocalization in metal doped graphene nanoribbons (GNRs) was found in the ascending order $\mathrm{Al}^{+} \rightarrow \mathrm{Cu}^{+} \rightarrow \mathrm{Co}^{+}$.

\subsection{Charge transfer numbers and electron-hole correlation plots}

The charge transfer numbers $\left(\mathbf{\Omega}_{\mathbf{A B}}\right)$ are calculated by summing up 1TDMs corresponding to the contributions from individual atoms/fragments $\mathbf{A}$ and $\mathbf{B}^{23,37}$ based on the following equation:

$$
\Omega_{\mathrm{AB}}=\frac{1}{2} \sum_{\mu \in A} \sum_{v \in B}\left[(\mathrm{DS})_{\mu \nu}(\mathbf{S D})_{\mu \nu}+\mathbf{D}_{\mu \nu}(\mathbf{S D S})_{\mu \nu}\right]
$$

where the summations run over the basis functions placed on $\mathbf{A}$ and $\mathbf{B}$, respectively. "S" is the overlap matrix between the basis functions. $\boldsymbol{\Omega}_{\mathbf{A B}}$ corresponds to the simultaneous probability that the hole is on fragment $\mathbf{A}$ while the electron is on $\mathbf{B}$ in the exciton picture. ${ }^{24}$ The weight of local excitations on fragment $\mathbf{A}$ is given by the diagonal element $\mathbf{\Omega}_{\mathbf{A A}}$ while charge transfer components are represented by off-diagonal elements $\boldsymbol{\Omega}_{\mathbf{A B}}(\mathbf{A} \neq \mathbf{B})$. The $\boldsymbol{\Omega}$ matrix can be visualized as a pseudocolor matrix plot yielding the so-called electron-hole (e-h) correlation plots. This representation, which is similar to 1TDM-based visualization techniques reported previously, ${ }^{28-30}$ provides a compact representation of excited state localization and charge transfer. In the present study the system was divided into four formal fragments corresponding to the different atom types $\mathbf{M}$ (metal), $\mathbf{H}$ (hydrogens), B (borons) and $\mathbf{N}$ (nitrogens). Thus, as shown in the top panel of Figure 4, 4x4 matrices are obtained representing the individual local and charge transfer contributions. The local contributions $(\mathrm{M} \rightarrow \mathrm{M}, \mathrm{H} \rightarrow \mathrm{H}, \mathrm{B} \rightarrow \mathrm{B}$, and $\mathrm{N} \rightarrow \mathrm{N})$ are located in the main diagonal (going from the lower left to the upper right) while the charge transfer contributions correspond to the off-diagonal elements. The weight of each individual type of excitation is encoded in grey scale.

Figure 4 illustrates the e-h correlation plots for the dominant excitations in the different MBNFs. To start with, in case of pure BNF, all the bright excitations $\mathrm{S}_{2}, \mathrm{~S}_{14}$ and $\mathrm{S}_{94}$ are dominated by $\mathrm{N} \rightarrow \mathrm{B}$ charge transfer while local $\mathrm{N} \rightarrow \mathrm{N}$ and $\mathrm{B} \rightarrow \mathrm{B}$ contributions are also visible for $\mathrm{S}_{14}$ and $\mathrm{S}_{94}$ transitions. For MBNF-Al, the block denoted by $\mathrm{N} \rightarrow \mathrm{B}$ is the darkest for all three dominant transitions $\left(\mathrm{S}_{51}, \mathrm{~S}_{61}\right.$ and $\left.\mathrm{S}_{77}\right)$ indicating majority charge transfer from nitrogen to boron. 
However, in addition, for $\mathrm{S}_{61}$ and $\mathrm{S}_{77}$ transitions, a charge transfer also occurs between $\mathrm{N}->\mathrm{H}$. The Al-atom does not play an important role in any of the investigated transitions. In case of MBNF-Co, $\mathrm{S}_{7}$ and $\mathrm{S}_{9}$ transitions are primarily characterized by a local excitation on the cobalt atom. In addition, slight charge transfer also occurs between $B \rightarrow M, M \rightarrow B$ and $N \rightarrow M$. In $\mathrm{S}_{69}$ and $\mathrm{S}_{96}$ transitions, the most prominent charge transfer occurs between $\mathrm{N}->\mathrm{B}\left(\mathrm{S}_{69}\right)$ and $\mathrm{M} \rightarrow \mathrm{B}\left(\mathrm{S}_{96}\right)$. For $\mathrm{S}_{96}$, slight charge transfers also occur between $\mathrm{B} \rightarrow \mathrm{B}, \mathrm{N} \rightarrow \mathrm{B}$ and $\mathrm{M} \rightarrow \mathrm{H}$. Lastly, the MBNF$\mathrm{Cu}$ is characterized by a mixed charge transfer. The $\mathrm{S}_{1}$ transition is characterized by an even mixture between the $\mathrm{B} \rightarrow \mathrm{M}$ and $\mathrm{M} \rightarrow \mathrm{B}$ charge transfers as well as the corresponding local contributions $(M \rightarrow M, B \rightarrow B)$. Further, the $S_{17}$ transition consists of $B \rightarrow H$ and $M \rightarrow H$ transfer, followed by $\mathrm{N} \rightarrow \mathrm{B}$ and $\mathrm{N} \rightarrow \mathrm{H}$ for $\mathrm{S}_{55}$ transition and then the $\mathrm{S}_{84}$ transition which is entirely characterized by $\mathrm{N} \rightarrow \mathrm{B}$ charge transfer. Interesting observation from our previous report ${ }^{31}$ on metal doped GNRs suggested a dominant charge transfer from metal to rest-of-graphene. However, in this study, we observed not very significant charge transfer (in comparison with $\mathrm{N}$ $>B$ ) from metal atom (upon absorption) for $\mathrm{S}_{96}, \mathrm{~S}_{9}$ (in MBNF-Co), and $\mathrm{S}_{1}, \mathrm{~S}_{17}$ (in MBNF-Cu) only. Also, local excitation within metal atom was also visible. Generally speaking, the charge transfer number analysis provides a consistent picture with the NTO analysis of Fig. 3 but yields a somewhat more compact description.

\begin{tabular}{|l|l|l|l|}
\hline$M->N$ & $H->N$ & $B->N$ & $N->N$ \\
\hline$M->B$ & $H->B$ & $B->B$ & $N->B$ \\
\hline$M->H$ & $H->H$ & $B->H$ & $N->H$ \\
\hline$M->M$ & $H->M$ & $B->M$ & $N->M$ \\
\hline
\end{tabular}

a)

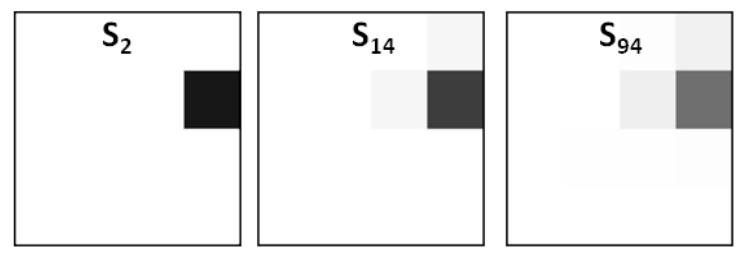

b)
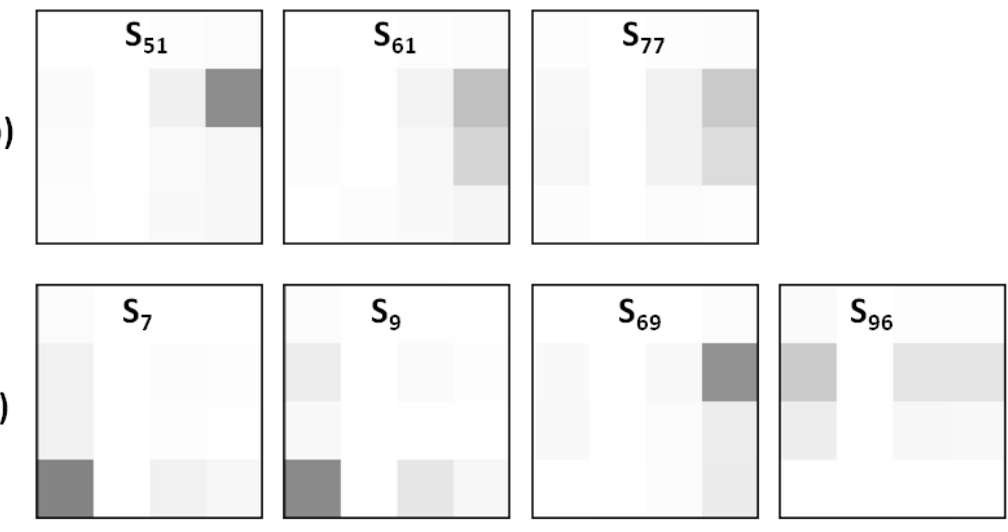
Fig. 4 Electron-hole correlation plots ( $4 X 4$ matrix) of dominant excitations $\left(\mathbf{S}_{\mathbf{0}}->\mathbf{S}_{\mathbf{n}}\right)$. Color scale is from 0 (White) to 1 (Black). a) Pure, b) MBNF-Al, c) MBNF-Co, and d) MBNF-Cu. The four fragments are $\mathbf{M}$ (metal atom) (except in a)), $\mathbf{H}$ (hydrogens), $\mathbf{B}$ (borons) and $\mathbf{N}$ (nitrogens). The $\mathbf{N} \rightarrow \mathbf{B}$ etc. symbols on the top image represent the charge transfer between the respective fragments.

\subsection{Electron-hole population analysis}

It is now intended to analyze the nature of the singlet transitions described in Table $\mathbf{1}$, based on the electron-hole (e-h) population analysis, which was obtained by using the post processing tool TheoDORE. These populations are given according to

$$
\boldsymbol{q}_{A}^{h}=\sum_{B} \mathbf{\Omega}_{\mathrm{AB}}
$$

for the hole population on fragment $\mathrm{A}$ and

$$
q_{B}^{e}=\sum_{A} \Omega_{\mathrm{AB}}
$$

for the electron population on fragment B. Here, again, analysis was done for fragments according to the atom types.

Firstly, in pure $\mathrm{BNF}$, the $\mathrm{S}_{0}->\mathrm{S}_{2}$ transition is of $\pi \pi^{*}$ type where the hole and electron are concentrated on the nitrogen atoms and boron atoms, respectively. It is well supported by the e-h population analysis with $93 \%$ hole character on nitrogen and $94 \%$ electron character on boron, indicating that it is and $\mathrm{N} \rightarrow \mathrm{B}$ excitation. Similarly, the $\mathrm{S}_{0^{-}}>\mathrm{S}_{14}$ transition is also of $\pi \pi^{*}$ nature (from $\mathrm{N} \rightarrow \mathrm{B}$ ) with $88 \%$ hole character on nitrogen and $88 \%$ electron character on boron. Lastly, the $\mathrm{S}_{0-}>\mathrm{S}_{94}$ transition is of $\pi \pi^{*}$ character, with $77 \%$ hole character on nitrogen and $76 \%$ electron character on boron. But, here the orbitals are more delocalized as compared to the other transitions.

In case of MBNF-A1, the $\mathrm{S}_{0^{-}}>\mathrm{S}_{51}$ transition is of $\pi \pi^{*}$ type, with $63 \%$ hole character on nitrogen and $61 \%$ electron character on boron. Interestingly, the hole character of boron has increased from $\sim 16 \%$ in pure form, to $\sim 20 \%$ in MBNF-A1. The $\mathrm{S}_{0-}>\mathrm{S}_{61}$ transition is a mixed transition, 
with $\pi \pi^{*}$ character ( $64 \%$ hole character on nitrogen and $43 \%$ electron character on boron), $15 \%$ electron character on aluminium atom indicating a $\mathrm{N}->\mathrm{Al}$ excitation and Rydberg character with $30 \%$ electron character on hydrogen atom. Lastly, the $\mathrm{S}_{0-}>\mathrm{S}_{77}$ transition is of $\pi \pi^{*}$ and Rydberg nature both, with the $52 \%$ hole character on nitrogen, $44 \%$ electron character on boron and $37 \%$ electron character on hydrogen.

Furthermore, for MBNF-Co, the $\mathrm{S}_{0}->\mathrm{S}_{7}$ transition is a local excitation, with $70 \%$ hole and $65 \%$ electron character both on the cobalt atom itself. The same is valid for the $\mathrm{S}_{0}->\mathrm{S}_{9}$ transition, with $65 \%$ and $68 \%$ hole and electron character. However, the $\mathrm{S}_{0-}>\mathrm{S}_{69}$ transition is of $\pi \pi^{*}$ type, with $71 \%$ hole on nitrogen and $53 \%$ electron on boron. The $\mathrm{S}_{0^{-}}>\mathrm{S}_{96}$ transition is an interesting one, where $\sim 24 \%$ hole character is on boron and nitrogen both and $43 \%$ on cobalt atom, and $60 \%$ electron character on boron. This indicates that excitation could be from $\mathrm{N}->\mathrm{B}\left(\pi \pi^{*}\right), \mathrm{N}->\mathrm{H}$ (Rydberg), Co- $>\mathrm{N}$ or $\mathrm{Co}->\mathrm{B}$.

Lastly, for the MBNF-Cu, the $\mathrm{S}_{0}->\mathrm{S}_{1}$ excitation is a local excitation due to $37 \%$ hole character and $48 \%$ electron both on the copper atom, or, $48 \%$ hole character and $38 \%$ electron both on the boron. The $\mathrm{S}_{0}->\mathrm{S}_{17}$ excitation could be from $\mathrm{Cu}->\mathrm{H}$ (Rydberg), B->B (local) or B->H (Rydberg), with hole $(35 \%)$ on Copper, electron $(70 \%)$ on hydrogen and $40 \%$ hole on boron. The $\mathrm{S}_{0-}>\mathrm{S}_{55}$ excitation is a combination of $\pi \pi^{*}(\mathrm{~N}->\mathrm{B})$ and Rydberg state $(\mathrm{N}->\mathrm{H})$ with $55 \%$ hole on nitrogen, $41 \%$ electron on boron and $34 \%$ electron on hydrogen. The last dominant transition $\mathrm{S}_{0}->\mathrm{S}_{84}$ is

purely of $\pi \pi^{*}$ character, with $65 \%$ hole character on nitrogen and $65 \%$ electron character on boron.

\subsection{Exciton sizes}

Root mean square electron-hole distances (d-eh) or exciton size determination is crucial in understanding the nature of excitons. Small exciton size indicates a tightly bound exciton, whereas large exciton size means a loosely bound exciton. It was noticed that a tightly bound exciton formed during absorption, leads to energy loss during the charge separation process ${ }^{38}$. This is crucial in photovoltaic devices. Hence a larger exciton is preferred, which would result in the lower exciton binding energy ${ }^{39,40}$. Exciton sizes (d-eh) are determined from the following equation, as described previously, ${ }^{25}+[$ S. A. Mewes, J.-M. Mewes, A. Dreuw, F. Plasser PCCP 2016, 18, 2548] and are derived from the TheoDORE package ${ }^{33}$. 


$$
\text { d-eh }=\sqrt{\frac{1}{\Omega} \sum_{A, B} \Omega_{A B} r^{2}{ }_{A B}}
$$

where $\boldsymbol{\Omega}_{\mathbf{A B}}$ are the charge transfer numbers, and $\mathbf{r}_{\mathbf{A B}}$ is the distance between the atoms $\mathbf{A}$ and $\mathbf{B}$, and $\boldsymbol{\Omega}$ the normalization factor is computed as the summation over all $\boldsymbol{\Omega}_{\mathbf{A B}}$. As opposed to the previous analysis, the summation goes over individual atoms in this case. At this point it should be noted that the employed pointcharge approximation cannot be directly translated to exciton binding energies, as this would encounter a division by zero. Therefore, only exciton sizes are computed. Table 2 shows the calculated values of the exciton sizes for the dominant singlet excitations.

Table 2. Exciton sizes $(\AA)$ corresponding to the dominant excitations for all the BNFs are listed.

\begin{tabular}{|l|c|c|}
\hline \multicolumn{1}{|c|}{ BNF } & $\begin{array}{c}\text { Excited } \\
\text { State }\left(\mathbf{S}_{\mathbf{n}}\right)\end{array}$ & $\begin{array}{c}\text { Exciton } \\
\text { size }(\boldsymbol{A})\end{array}$ \\
\hline \multirow{3}{*}{ Pure } & $\mathbf{S}_{\mathbf{2}}$ & 5.68 \\
& $\mathbf{S}_{\mathbf{1 4}}$ & 5.20 \\
MBNF-Al & $\mathbf{S}_{\mathbf{9 4}}$ & 5.01 \\
& $\mathbf{S}_{\mathbf{5 1}}$ & 4.70 \\
& $\mathbf{S}_{\mathbf{6 1}}$ & 5.0 \\
& $\mathbf{S}_{\mathbf{7 7}}$ & 5.52 \\
\hline \multirow{3}{*}{ MBNF-Co } & $\mathbf{S}_{\mathbf{7}}$ & 2.35 \\
& $\mathbf{S}_{\mathbf{9}}$ & 2.18 \\
& $\mathbf{S}_{\mathbf{6 9}}$ & 5.48 \\
& $\mathbf{S}_{\mathbf{9 6}}$ & 5.31 \\
\hline \multirow{3}{*}{ MBNF-Cu } & $\mathbf{S}_{\mathbf{1}}$ & 2.82 \\
& $\mathbf{S}_{\mathbf{1 7}}$ & 4.76 \\
& $\mathbf{S}_{\mathbf{5 5}}$ & 5.75 \\
& $\mathbf{S}_{\mathbf{8 4}}$ & 4.56 \\
\hline
\end{tabular}

Materials with a large exciton radius are preferred in the photovoltaic application. In this study, the pure BNF would be the preferred candidate due to its large exciton sizes as compared to metal doped BNFs. The exciton sizes of all the BNFs for the dominant transitions range within 2.18-5.75A. Exciton sizes have been directly related to the delocalization of electron density ${ }^{41}$, even though it should be noted that this interpretation changes for strongly correlated exciton states $^{26}$. In the present study, we have used the exciton size to study the extent of electron density delocalization. For the pure BNF, the $\mathrm{S}_{94}$ transition is the one with the least electron delocalization as compared to $\mathrm{S}_{2}$ and $\mathrm{S}_{14}$ transitions. It is also well supported through NTO 
analysis where the spread of exciton wavefunction is minimal from hole to electron NTO. In case of MBNF-A1, $\mathrm{S}_{77}$ transition has the largest exciton radius (5.52 $\AA$ ) indicating the highest electron delocalization compared to the $\mathrm{S}_{51}$ and $\mathrm{S}_{61}$ transitions. This is well correlated with the NTO analysis where the spread of electron density is larger on absorption. Next, in MBNF-Co, $\mathrm{S}_{7}$ is characterized by the lowest exciton radius of $2.35 \AA$. This is well explained by observing NTOs where the electron density is floating around the metal atom itself, upon absorption resulting in the least electron delocalization. On the contrary, the $\mathrm{S}_{96}$ transition is featured with the highest electron delocalization. Lastly, in MBNF-Cu, the $\mathrm{S}_{1}$ transition is characterized by the least delocalization (exciton size $=2.82 \AA$ ) due to the minimal movement of the electron density from the metal atom. However, the $\mathrm{S}_{55}$ excitation demonstrates the highest delocalization on absorption, with the electron cloud spanning a larger area.

\section{Conclusion}

Electronic excitations upon absorption in boron nitride flakes (BNFs) both in pure $\left(\mathrm{B}_{15} \mathrm{~N}_{15} \mathrm{H}_{14}\right)$ and neutral single-metal-atom doped $\left(\mathrm{B}_{15} \mathrm{~N}_{14} \mathrm{H}_{14}-\mathrm{X}\right.$, where $\mathrm{X}=\mathrm{Al}, \mathrm{Co}$, and $\left.\mathrm{Cu}\right)$ forms were studied with the help of TDDFT calculations and transition density matrix based analysis. All the BNFs absorb in the UV range. However, strong red shifts were seen for all metal doped cases, with the strongest shift for MBNF-Co. Different analysis methods were applied to illustrate different aspects of the excitations. The natural transition orbital analysis was applied to illustrate the absorption process in the orbital picture and helped in understanding the electron delocalization upon absorption. Charge transfer plots were analyzed and it was found that in pure $\mathrm{BNF}$, charge transfer is primarily from $\mathrm{N}->\mathrm{B}$, however, a more diverse charge transfer occurs in metal doped forms, and in the case of MBNF-Cu and MBNF-Co strong involvement of the metal atom was found. Electron-hole population analysis was done to determine the nature of excitations ( $\pi \pi^{*}$, Rydberg or local). Lastly, the exciton sizes were calculated and found to be in the range 2.18-5.75 $\AA$ for all the BNFs, and were used in the electron delocalization analysis. Generally, a consistent description was obtained between the various analysis methods. This study illustrates how strongly the excited state properties in BNF can vary upon doping and improves the understanding of electronic excitations in boron-nitride nanomaterials, which would be beneficial in photovoltaic applications. Furthermore, it serves as an exemplary investigation of how excited state analysis methods can be applied to maximize the insight gained from excited state computations performed in extended systems. In future, we further wish to study the effect of size and shape of BN flakes on the electronic and optical properties. 



\section{Acknowledgements}

SC acknowledges the National PARAM Supercomputing Facility (NPSF) of C-DAC, Pune, India for providing the cluster computing facility. FP acknowledges support from the VSC Research Center funded by the Austrian Federal Ministry of Science, Research, and Economy (bmwfw). 


\section{REFERENCES}

1. N. Koi, T. Oku, and M. Nishijima, Solid State Commun. 2005, 136, 342.

2. K.P. Loh, M. Lin, M. Yeadon, C. Boothroyd and Z. Hu, Chem Phys Lett. 2004, 387, 40.

3. D. Golberg, F.F. Xu and Y. Bando, Appl Phys A 2003, 76, 479.

4. J. Beheshtian, A.A. Peyghan Z. and Bagheri, Sens Actuators B 2012, 846, 171-172.

5. Q. Sun, Z. Li, D.J. Searles, Y. Chen, G. Lu and A. Du, J. Am. Chem. Soc. 2013, 135, 8246-8253.

6. S. Hagiwara, H. Goto, C. Hu and K. Watanabe, JPS Conf. Proc., 2014, 012072.

7. L. Koponen, L. Tunturivuori, M.J. Puska and R.M. Nieminen, J. Chem. Phys. 2007, 126, 214306.

8. M. Sajjad, W.M. Jadwisienczakb and P. Feng, Nanoscale 2014, 6, 4577-4582.

9. J.H. Mokkath and U. Schwingenschlogl, J. Mater. Chem. C 2014, 2, 8322-8327.

10. R.Z. Ma, Y. Bando, H.W. Zhu, T. Sato, C.L. Xu and D.H. Wu, J. Am. Chem. Soc. 2002, 124, 76727673.

11. C.C. Tang, Y. Bando, X.X. Ding, S.R. Qi, and D. Golberg, J. Am. Chem. Soc. 2002, 124, 14 550-14 551.

12. C.R.A. Catlow, Z.X. Guo, M. Miskufova, S.A. Shevlin, A.G.H. Smith, A.A. Sokol, A. Walsh, D.J. Wilson and S.M. Woodley, Phil. Trans. R. Soc. A 2010, 368, 3379-3456.

13. Z. Zhang, Z. Geng, D. Cai, T. Pan, Y. Chen, L. Dong, L. and T. Zhou, Physica E 2015, 56, $24-29$.

14. D. Ma, Z. Lu, W. Ju, and Y. Tang, J. Phys.: Condens. Matter 2012, 24, 145501-145508.

15. S. Chopra, Molecular Physics 2016, 114, 13, 1-7.

16. E. Runge and E.K.U. Gross, Phys. Rev. Lett. 1984, 52, 997.

17. M.E. Casida, in: D.P. Chong (Ed.), Recent Advances in Density Functional Methods, vol. 1, World Scientific, Singapore, 1995.

18. A. Dreuw and M. Head-Gordon, Chem. Rev. 2005, 105, 4009.

19. M.A.L. Marques, C.A. Ullrich, F. Nogueira, A. Rubio, K. Burke and E.K.U. Gross(Eds.), TimeDependent Density Functional Theory, Springer-Verlag, 2006.

20. J.-L. Brédas, J. Cornil, D. Beljonne, D. A. dos Santos, and Z. Shuai, Acc. Chem. Res. 1999, 32, 267.

21. N. Kirova, Polym. Int. 2008, 57, 678.

22. D. Abramavicius, B. Palmieri, D.V. Voronie, F. Šanda and S. Mukamel, Chem. Rev. 2009, 109, 2350.

23. F. Plasser and H. Lischka, J. Chem. Theory Comput.2012, 8, 2777.

24. F. Plasser, M. Wormit, and A. Dreuw, The Journal of Chemical Physics 2014, 141, 024106.

25. S. A. Bappler, F. Plasser, M. Wormit, and A. Dreuw, Physical Review A 2014, 90, 052521.

26. F. Plasser, B. Thomitzni, S.A. Bäppler, J. Wenzel, D.R. Rehn, M. Wormit and A. Dreuw, J. Comp. Chem 2015, 36, 1609.

27. R. L. Martin, J. Chem. Phys. 2003, 118, 4775.

28. E. Zojer, P. Buchacher, F. Wudl, J. Cornil, J. Ph. Calbert, J. L. Brédas and G. Leising, J. Chem. Phys. 2000, 113, 10002.

29. J. Rissler, H. Bäßler, F. Gebhard and P. Schwerdtfeger, Phys. Rev. B 2001, 64, 045122.

30. S. Tretiak and S. Mukamel, Chem. Rev. 2002, 102, 3171-3212.

31. S. Chopra, RSC Advances 2016, 6, 20565 - 20570.

32. A. A. Granovsky, Firefly version 8.1.0., http://classic.chem.msu.su/gran/firefly/index.html

33. F. Plasser, TheoDORE: A Package for Theoretical Density, Orbital Relaxation, and Exciton Analysis, Available at http://theodore-qc.sourceforge.net/.

34. N. M. O'Boyle, A. L. Tenderholt and K. M. Langner, J. Comp. Chem. 2008, 29, 5, 839-845. 35. Jmol 13.0.8, Available at http://sourceforge.net/projects/jmol. 
36. M. E. Casida, Journal of Molecular Structure: THEOCHEM 2009, 914, 3-18.

37. A. V. Luzanov and O. A. Zhikol, Int. J. Quant. Chem. 2010, 110, 902.

38. G. Dennler, M. C. Scharber, and C. J. Brabec, Advanced Materials 2009, 21, 1323.

39. K. Hummer and C. Ambrosch-Draxl, Physical Review B 2005, 71, 081202.

40. M. Knupfer, Applied Physics A: Materials Science \& Processing 2003, 77, 623.

41. S. Tretiak, K. Igumenshchev, and V. Chernyak, Physical Review B 2005, 71, 3. 